\title{
Center Volume Is Associated With Outcome After Pancreas Transplantation Within the Eurotransplant Region
}

\author{
Wouter Kopp, MD, ${ }^{1,2}$ Marieke van Meel, BSc, ${ }^{2}$ Hein Putter, PhD, ${ }^{3}$ Undine Samuel, MD, ${ }^{2}$ \\ Helmut Arbogast, MD, PhD, ${ }^{4}$ Wolfgang Schareck, MD, PhD, ${ }^{5}$ Jan Ringers, $\mathrm{MD},{ }^{1}$ and Andries Braat, MD, $\mathrm{PhD}^{1}$
}

Background. Outcome after surgery depends on several factors, among these, the annual volume-outcome relationship. This might also be the case in a highly complex field as pancreas transplantation. No study has investigated this relationship in a European setting. Methods. All consecutive pancreas transplantations from January 2008 until December 2013 were included. Donor-, recipient-, and transplant-related factors were analyzed for their association with patient and graft survivals. Centers were classified in equally sized groups as being low volume ( $<5$ transplantations on average each year in the 5 preceding years), medium volume (5-13/year), or high volume ( $\geq 13 /$ year). Results. In the study period, 1276 pancreas transplantations were included. Unadjusted 1 -year patient survival was associated with center volume and was best in high volume centers, compared with medium and low volume: $96.5 \%, 94 \%$ and $92.3 \%$, respectively $(P=0.017)$. Pancreas donor risk index (PDRI) was highest in high volume centers: 1.38 versus 1.21 in medium and 1.25 in low volume centers $(P<0.001)$. Pancreas graft survival at 1 year did not differ significantly between volume categories: $86 \%, 83.2 \%$, and $81.6 \%$, respectively $(P=0.114)$. After multivariate Cox-regression analysis, higher PDRI (hazard ratio [HR], 1.60; $P<0.001$ ), retransplantation (HR, 1.91; $P=0.002)$, and higher recipient body mass index $(H R, 1.04 ; P=0.024)$ were risk factors for pancreas graft failure. High center volume was protective for graft failure (HR, 0.70 ; $P=0.037)$ compared with low center volume. Conclusion. Patient and graft survival after pancreas transplantation are superior in higher volume centers. High volume centers have good results, even though they transplant organs with the highest PDRI.

(Transplantation 2017;101: 1247-1253)

ancreas transplantation is the only definitive treatment for patients with type 1 diabetes mellitus. This can be as a simultaneous pancreas kidney transplantation (SPK) in case of end-stage renal disease (ESRD) or as a solitary pancreas transplant (pancreas after kidney [PAK], pancreas

Received 6 January 2016. Revision received 19 April 2016.

Accepted 20 April 2016.

${ }^{1}$ Division of Transplantation, Department of Surgery, Leiden University Medical Center, Leiden, The Netherlands.

${ }^{2}$ Eurotransplant International Foundation, Leiden, The Netherlands.

${ }^{3}$ Department of Statistics, Leiden University Medical Center, Leiden, The Netherlands.

${ }^{4}$ Department of General, Visceral, Transplant, Vascular and Thoracic Surgery, University of Munich-Grosshadern Medical Centre, Munich, Germany.

${ }^{5}$ University Hospital Rostock, Rostock, Germany.

H.A., W.S., and J.R. represent the Eurotransplant Pancreas Advisory Committee.

W.H.K. and J.R. designed the study. M.M. and U.S. collected the data. H.P. and W.H.K. performed the statistical analysis. W.H.K., U.S., and A.E.B. wrote the article. All authors participated in critical appraisal and revision of the article.

The authors declare no funding or conflicts of interest.

Correspondence: W.H. Kopp, MD, Division of Transplantation, Department of Surgery, Leiden University Medical Center, Albinusdreef 22333 ZA Leiden, The Netherlands. (w.h.kopp@/umc.nl).

Supplemental digital content (SDC) is available for this article. Direct URL citations appear in the printed text, and links to the digital files are provided in the HTML text of this article on the journal's Web site (www.transplantjournal.com).

Copyright @ 2016 Wolters Kluwer Health, Inc. All rights reserved.

ISSN: 0041-1337/17/10106-1247

DOI: 10.1097/TP.0000000000001308 transplant alone [PTA]) in case of life-threatening hypoglycemic unawareness. ${ }^{1-3}$ Even though the number of patients on the waiting list is relatively stable since 2009 , optimal usage of scarce number of potential pancreas allografts is still highly important. ${ }^{4}$ Apart from donor, recipient and transplant factors influencing outcome after transplantation, 5,6 center factors may also play a significant role.

The Dutch Institute for Clinical Auditing has been working on valid outcome measures in 18 domains of healthcare, most of them in oncological surgery. Eurotransplant is a nonprofit organization that facilitates patient-oriented allocation and cross-border exchange of deceased donor organs. Active for transplant centers and their associated tissue typing laboratories and donor hospitals in 8 countries, Eurotransplant ensures an optimal use of donor organs. To be able to develop allocation policies based on state-of-the-art medical knowledge, Eurotransplant collects donor, recipient, and center data, as well as outcome data after transplantation. Information on center-related outcome, provided that they represent valid and useful outcome measures, should be publically available: to centers, to improve their results; to patients, to make a wellfounded decision on a preferred center; and to politicians, to design legitimate healthcare policies. This information can be derived from organizations, such as the Dutch Institute for Clinical Auditing or Eurotransplant or from single-center reports.

With this information, efforts are being put into concentrating "high complex, low volume" care in The Netherlands. ${ }^{7}$ Especially oncology care is subject of this ongoing change. Transplantation has been the subject of concentration of care 
by the government longer and especially pancreas transplantation, with currently only 2 of 8 transplant centers with an active pancreas transplantation program. The question rises whether this concentration is justified and if the volume outcome relationship also exists in the field of pancreas transplantation, as has been stated before. ${ }^{8,9}$ Recently, a German study advocated for an extensive analysis of volume-outcome after transplantation. ${ }^{10}$ In 2014, within Eurotransplant there were 37 centers with an active pancreas transplant program, performing a total of 199 vascularized pancreas transplants, thus averaging an annual number of pancreas transplantations of a little over 5 each year. ${ }^{4}$

The aim of this study is to investigate the effect of center volume on outcome after pancreas transplantation in the Eurotransplant region.

\section{Design}

All consecutive vascularized pancreas transplantations that were performed in Eurotransplant centers from January 1, 2008, until December 31, 2013, were analyzed. Donor, procurement, recipient, and transplant data that were derived from the standard Eurotransplant database are shown in Table 1. Follow-up data were collected through the Eurotransplant registry. The Eurotransplant registry data were extracted at October 6, 2015. Graft survival was death censored. A frequently used definition of graft failure is that graft failure has occurred, when the recipient had returned to exogenous insulin therapy. This was the definition that the authors applied to all patients that were transplanted at the Leiden University Medical Center. For all other centers, it was unknown which definition was used, so the definition of pancreas graft failure was left up to the discretion of the transplant centers. When graft failure and death occurred at the same day or a graft had not been reported as failed before recipient death, this was not considered graft failure, and these cases were censored. The procurement surgeon determined organ quality (good, acceptable, poor) based on macroscopic evaluation; however, exact criteria were unknown.

Center volume for each year was defined as the total transplant volume of the 5 preceding years, based on standard Eurotransplant data reports (ie, factor center volume for 2008 was based on average volume from 2003 to 2007, for 2009 based on 2004 to 2008 , and so on). ${ }^{11}$ Volume calculations were not performed for center before their entry in the Eurotransplant collaboration. Croatia entered Eurotransplant in 2007, therefore, only transplants in 2013 (volume based on 2008-2012) were included in the center volume-survival analysis. Hungary entered in 2013, so was excluded from the center volume-survival analysis. Three equally sized groups were determined (low, medium, and high volume), based on the total volume in the 5 preceding years. Multiorgan transplants were only used to compute the total volume and were excluded from further analysis. Data in all 3 categories were pooled in order not to compromise recipient privacy and in order to not be able to identify individual centers.

\section{Statistical Analysis}

Differences between different volume categories were displayed using pooled sample mean and SE. $P$ values were calculated using 1-way analysis of variance. Missing values were imputed using 20 imputation rounds. Missing survival data were not imputed. Survival analysis for categorical variables was done using Kaplan-Meier estimates and groups were compared using Log-rank tests. Continuous variables were analyzed using Cox proportional hazard models after testing of the proportional hazards assumption. ${ }^{12} P$ values less than 0.05 were considered statistically significant. All significant factors from univariate survival analysis, as well as factors that were different among volume groups were entered in multivariate Cox proportional hazards model. To account for clustering of the data, robust sandwich estimates of the standard errors were used in multivariate analysis. ${ }^{13}$ Only complete cases after multiple imputations were analyzed.

\section{Missing Data Imputation}

Recipient weight $(6.2 \%)$, recipient height $(6.2 \%)$, and pancreas cold ischemia $(25.4 \%)$ had missing values. Variables that were included in the imputation model were: donor age, sex, weight, height, body mass index (BMI), cause of death, creatinine, DBD versus DCD, pancreas donor risk index (PDRI), and donor country; recipient age, sex, weight, height, dialysis type, waiting time; pancreas cold ischemia time in minutes and hours, total pancreas cold ischemia time (hours), transplant type (SPK, PAK, PTA), center volume, warm ischemic period, transplant center, transplant year, organ quality, perfusion solution. Warm ischemic time, PDRI, creatinine, amylase, lipase, sodium, transplant center, donor country, perfusion solution, and organ quality were used as indicators only. Imputation method was automatically selected by SPSS (SPSS version 22, IBM, North Castle, NY) based on patterns of missing value analysis. To reduce sampling variability from the imputations, 20 imputation rounds were performed. ${ }^{14}$ Results of multiple imputations are shown in Table 2. Recipient BMI and PDRI were calculated based on the imputed values.

\section{RESULTS}

In the study period (January 2008 to December 2013), 1276 pancreas transplantations were included in the study. There were 1148 (90\%) SPK transplantations, 84 (6.6\%) PAK transplantation, and $44(3.4 \%)$ PTA transplantations. During the study and follow-up period, $122(9.6 \%)$ patients were reported deceased and $256(20.1 \%)$ grafts were reported as failed (death-censored). Mean duration of followup was 3.2 years. Mean pancreas donor risk index was 1.27. Demographics are shown in Table 1.

\section{Patient and Pancreas Graft Survival}

Overall patient survival at 180 days, 1 year, and 3 years was $95.4 \%, 94.1 \%$, and $91.2 \%$, respectively. Patient death was associated with higher recipient age (hazard ratio [HR], 1.03; $P=0.006$ ).

Pancreas graft survival (death-censored) at 180 days, 1 year, and 3 years was $85.3 \%, 83.7 \%$, and $78.8 \%$, respectively. Pancreas graft failure was associated with higher donor age $(P=0.006)$, higher donor BMI $(P=0.036)$, higher PDRI $(P=0.007)$, and high recipient BMI $(P=0.027)$, retransplantation $(P<0.001)$ and the use of histidine tryptophan ketoglutarate $(\mathrm{HTK})$ as perfusion solution $(P=0.036)$. Simultaneous pancreas kidney transplantation $(P<0.001)$ was protective in univariate analysis. Results of univariate analysis on factors associated with pancreas graft failure are shown in Table 1. Year of transplant was not associated with pancreas graft survival $(P=0.69)$. 


\section{TABLE 1.}

Demographics, univariate analysis of association with pancreas graft survival ${ }^{a}$

\begin{tabular}{|c|c|c|c|c|}
\hline & $\mathrm{n}$ & $\%$ & $P$ & $x^{2}$ \\
\hline Donors & 1276 & 100 & & \\
\hline $\mathrm{Sex}^{b}$ (male) & & & 0.150 & 2.070 \\
\hline Male & 678 & 53.1 & & \\
\hline Female & 598 & 46.9 & & \\
\hline Cause of death ${ }^{b}$ & & & 0.076 & 8.460 \\
\hline Cerebrovascular accident & 624 & 48.9 & & \\
\hline Trauma & 497 & 38.9 & & \\
\hline Circulational/anoxia & 115 & 9 & & \\
\hline CNS tumor & 7 & 0.5 & & \\
\hline Other & 33 & 2.6 & & \\
\hline Donor type $^{b}$ & & & 0.387 & 0.749 \\
\hline DBD & 1268 & 99.4 & & \\
\hline DCD & 8 & 0.6 & & \\
\hline
\end{tabular}

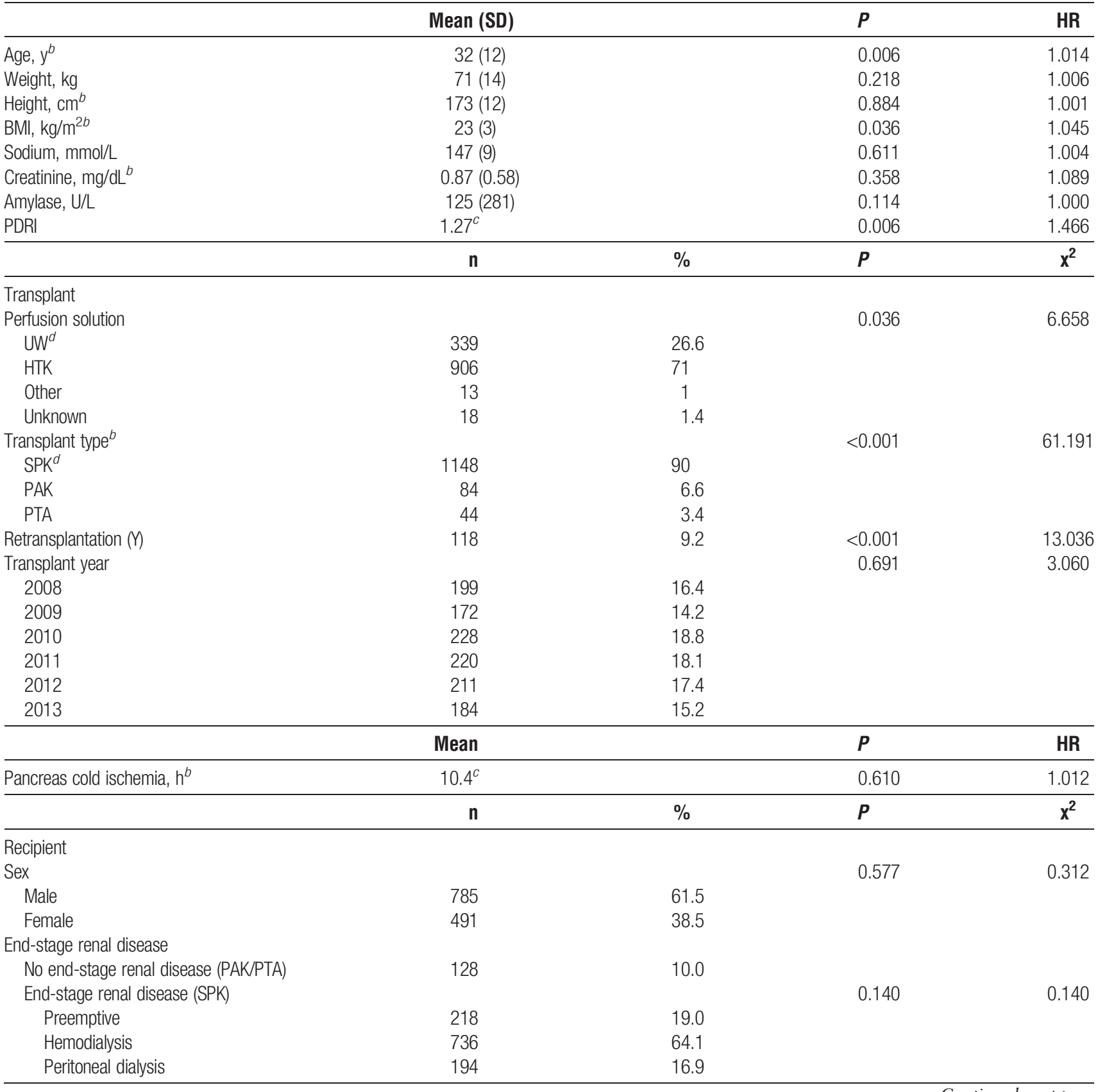




\begin{tabular}{|c|c|c|c|}
\hline & Mean (SD) & $P$ & HR \\
\hline Age, y & $44(9)$ & 0.487 & 0.995 \\
\hline BMI, $\mathrm{kg} / \mathrm{m}^{2}$ & $24^{c}$ & 0.025 & 1.038 \\
\hline Waiting time, y & $1.15(1.3)$ & 0.970 & 0.998 \\
\hline
\end{tabular}

${ }^{a}$ Kaplan-Meier estimates (Log rank Mantel-Cox) for categorical variables. Cox proportional hazards for continuous variables.

${ }^{b}$ PDRI factor.

${ }^{c}$ Based on imputed data.

${ }^{d}$ Favorable factor.

In a separate subgroup analysis of recipients with ESRD (SPK recipients), the influence of dialysis modality (either preemptive transplantation, peritoneal dialysis or hemodialysis) was analyzed for the association with patient and graft survival. In this analysis, patient $(P=0.235)$ and graft survivals $(P=0.140)$ were not associated with dialysis technique.

\section{Analysis of Center Volume on Outcome}

For $1214(95.1 \%)$ transplantations, center volume was calculated. Nine transplantations $(0.7 \%)$ were from Hungary and $53(4.2 \%)$ from Croatia, and these were excluded, because they had too few preceding years in Eurotransplant. Low volume centers ( $<25$ transplantation $/ 5$ preceding years) performed $396(32.6 \%)$ transplantations, $425(35 \%)$ were performed in medium volume (25-64 transplantation/5 years) centers and $393(32.4 \%$ ) in high volume ( $\geq 65$ transplantation/5 years) centers. An overview of number of transplantations in each year by center category is shown in Table S1 (SDC, http://links.lww. com/TP/B303). Center demographics are shown in Table 3. The pooled sample mean PDRI of donors transplanted in different categories differed significantly: 1.25 in low volume centers, 1.21 in medium volume centers, and 1.38 in high volume centers $(P<0.001)$. Post hoc testing (Bonferroni corrections) showed that PDRI only differed between high versus low $(P<0.001)$ and high versus medium $(P<0.001)$, not low versus medium $(P=0.316)$. High volume centers transplanted patients with ESRD more frequently in a preemptive setting, compared with low and medium volume $(P<0.001)$. Mean time from waiting list registration to transplantation was not significantly different in 3 volume categories (Table 3 ). The proportional hazards assumption was not violated $(P=0.350)$.

Patients transplanted in high volume centers had longest patient survival $(P=0.017)$ (Figure 1A). Other than age and center volume, no factors were significantly associated with patient survival in univariate analysis. After correcting for recipient age (HR, 1.04; 95\% confidence interval [95\% $\mathrm{CI}], 1.02-1.06 ; P=0.001$ ) in a multivariate Cox regression analysis, high volume $(\mathrm{HR}, 0.51 ; 95 \% \mathrm{CI}, 0.32-0.81$,
$P=0.004)$ but not medium volume (HR, 0.65; 95\% CI, $0.42-1.00 ; P=0.052$ ) was protective compared with low volume. One hundred twelve cases $(8.8 \%)$ were excluded due to missing follow-up data or because they were performed in the first years of Croatian or Hungarian membership of Eurotransplant.

In univariate analysis, graft survival was not significantly different among the 3 categories $(P=0.11)$ (Figure 1B). Higher PDRI (HR, 1.60; $P<0.001)$, retransplantation (HR, 1.91; $P=0.002)$, and higher recipient BMI (HR, 1.04; $P=0.024$ ) were independent risk factors for pancreas graft failure after multivariate Cox regression analysis. Perfusion with University of Wisconsin (UW) solution was not protective after multivariate analysis, compared with HTK $(P=0.111)$ or other solutions $(P=0.739)$. Higher center volume was associated with a lower risk of pancreas graft failure. This effect was statistically significant for low versus high volume (HR, 0.70; $P=0.037$ ), but not for low versus medium volume (HR, 0.89; $P=0.562$ ). Results of multivariate analyses are shown in Table $4 a$. One hundred thirty $(10.2 \%)$ cases were excluded from multivariate analysis due to missing follow-up data or because they were performed in the first years of Croatian or Hungarian membership of Eurotransplant.

In a separate subgroup analysis (Table $4 \mathrm{~b}$ ) with only SPK transplant included, PDRI, volume category, and perfusion solution (significant factors from univariate analysis), recipient BMI, and dialysis category were included in multivariate analysis. In this multivariate analysis, high PDRI was associated with graft failure (HR, 1.94; $P<0.001)$. High volume, as compared to low volume, was protective for graft failure (HR, 0.69; $P=0.032$ ), whereas medium volume was not (HR, 0.91; $P=0.696)$. The use of HTK was associated with a higher risk of graft failure compared with UW (HR, 1.56, $P=0.021$ ). Whether a recipient was transplanted preemptively or while on dialysis was not associated with pancreas graft survival. Of all 1148 SPK transplantations, 119 $(10.4 \%)$ were excluded from multivariate analysis due to missing follow-up data or because they were performed in

\section{TABLE 2.}

\section{Imputation of missing data}

\begin{tabular}{|c|c|c|c|c|c|c|}
\hline & \multicolumn{3}{|c|}{ Original data } & \multicolumn{3}{|c|}{ Imputed data ${ }^{a}$} \\
\hline & $\mathbf{n}$ & $\%$ missing & Mean (SEM) & $\mathbf{n}$ & $\%$ Missing & Mean (SEM) \\
\hline Recipient height, $\mathrm{cm}$ & 1198 & 6.2 & $172(0.26)$ & 1276 & 0 & $172(0.26)$ \\
\hline Recipient weight, kg & 1198 & 6.2 & $72(0.39)$ & 1276 & 0 & $72(0.40)$ \\
\hline Pancreas CIT, h & 952 & 25.4 & $10.4(0.09)$ & 1276 & 0 & $10.4(0.08)$ \\
\hline
\end{tabular}

a 20 rounds of multiple imputations. 


\section{TABLE 3.}

Demographics in center categories

\begin{tabular}{|c|c|c|c|c|}
\hline & Low volume & Medium volume & High volume & $P^{a}$ \\
\hline $\mathrm{n}$ & $396(32.6 \%)$ & 425 (35\%) & $393(32.4 \%)$ & \\
\hline PDRI & $1.25(0.41)$ & $1.21(0.41)$ & $1.38(0.46)$ & $<0.001$ \\
\hline \multicolumn{5}{|l|}{ PDRI factors } \\
\hline Donor age, y & $33(11)$ & $30(12)$ & $35(13)$ & $<0.001$ \\
\hline Donor BMl, kg/m² & $23.6(2.8)$ & $22.9(3.2)$ & $23.3(2.9)$ & 0.005 \\
\hline Pancreas CIT, h & $9.7(2.6)$ & $10.4(3.1)$ & $11.2(2.6)$ & $<0.001$ \\
\hline SPK transplantation & $361(91.2 \%)$ & $375(88.2 \%)$ & $353(89.8 \%)$ & 0.019 \\
\hline Cause of death (stroke) & $196(49.5 \%)$ & $194(45.6 \%)$ & $210(53.4 \%)$ & 0.252 \\
\hline DCD & $0(0 \%)$ & $0(0 \%)$ & $8(2 \%)$ & $<0.001$ \\
\hline Recipient age, y & $44(8.7)$ & $44(8.6)$ & $44(8.7)$ & 0.660 \\
\hline Recipient BMI, kg/m² & $24.2(4.2)$ & $24.1(3.8)$ & $24.2(3.8)$ & 0.593 \\
\hline Sensitized & & & & 0.177 \\
\hline 6-80\% PRA & $15(4 \%)$ & $28(6.9 \%)$ & $21(5.5 \%)$ & \\
\hline$>80 \%$ PRA & $3(0.8 \%)$ & $0(0 \%)$ & $3(0.8 \%)$ & \\
\hline Waiting time, $\mathrm{d}$ & $586(434)$ & $649(497)$ & $583(532)$ & 0.087 \\
\hline Retransplantations & $32(8.1 \%)$ & $46(10.8 \%)$ & $40(10.2 \%)$ & 0.387 \\
\hline End-stage renal disease (SPK) & & & & $<0.001$ \\
\hline Preemptive & $61(16.9 \%)$ & $54(14.4 \%)$ & $101(28.6 \%)$ & \\
\hline Hemodialysis & $243(67.3 \%)$ & $262(69.9 \%)$ & $192(54.4 \%)$ & \\
\hline Peritoneal dialysis & 57 (15.8\%) & $59(15.7 \%)$ & $60(17.0 \%)$ & \\
\hline
\end{tabular}

${ }^{a}$ One-way ANOVA for continuous variables (mean, SD), $\mathrm{x}^{2}$ for categorical variables (n, \%).

${ }^{b}$ Low volume ( $<5$ transplantations/year), medium volume (5-13/year) or high volume ( $\geq 13 /$ year).

A

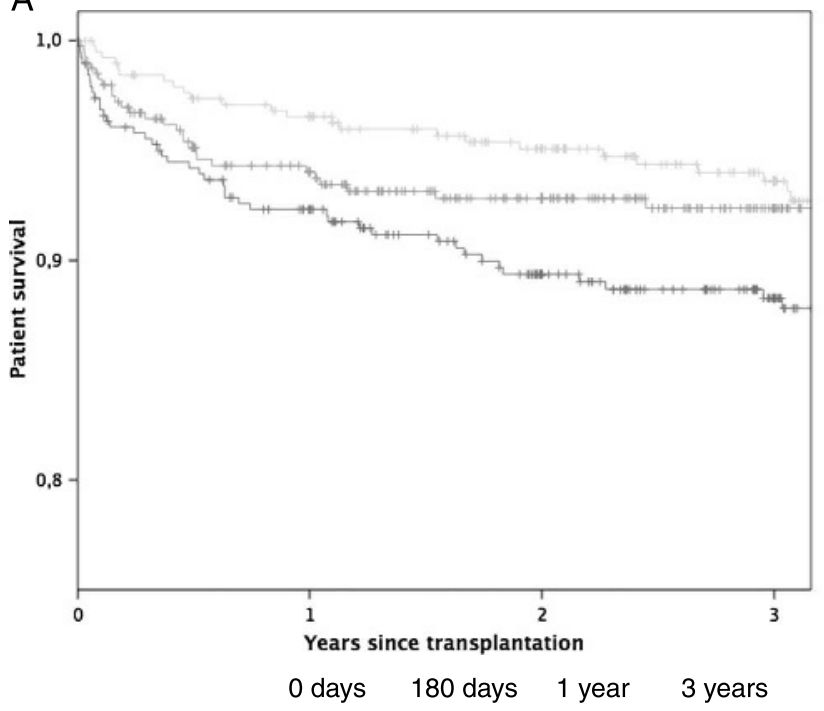

Low volume

$\begin{array}{lcccc}\text { N at risk } & 382 & 353 & 332 & 216 \\ \text { Patient survival } & 100 \% & 94.2 \% & 92.3 \% & 88.3 \%\end{array}$

Medium volume

$\begin{array}{lcccc}\text { N at risk } & 399 & 353 & 329 & 170 \\ \text { Patient survival } & 100 \% & 95.1 \% & 94.0 \% & 92.4 \%\end{array}$

\section{High volume}

$\begin{array}{lcccc}\text { N at risk } & 382 & 360 & 346 & 236 \\ \text { Patient survival } & 100 \% & 97.4 \% & 96.5 \% & 93.6 \%\end{array}$

B

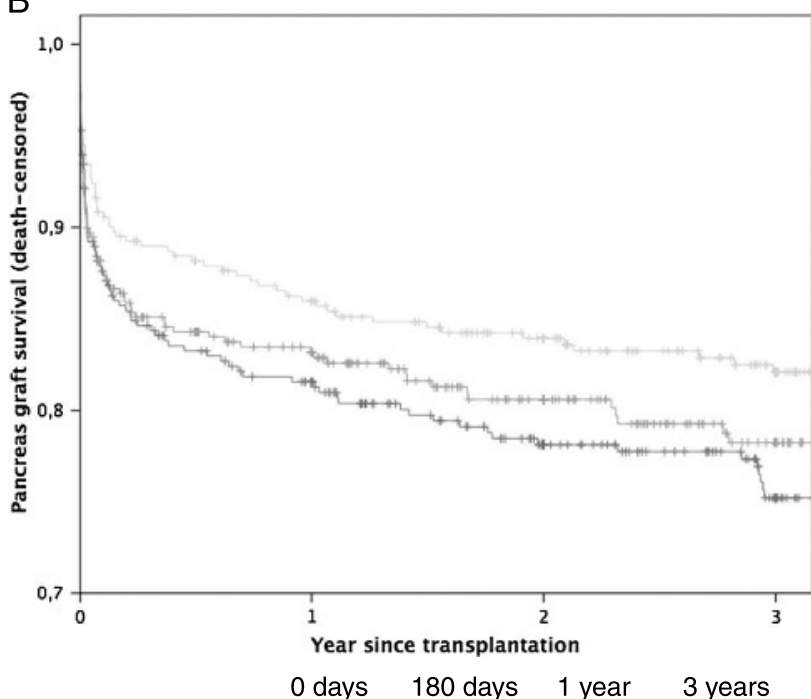

Low volume

$\begin{array}{lcccc}\text { N at risk } & 382 & 300 & 280 & 170 \\ \text { Graft survival } & 100 \% & 83.3 \% & 81.6 \% & 75.2 \%\end{array}$

Medium volume

$\begin{array}{lcccc}\text { N at risk } & 399 & 310 & 284 & 139 \\ \text { Graft survival } & 100 \% & 84.3 \% & 83.2 \% & 78.2 \%\end{array}$

\section{High volume}

$\begin{array}{lcccc}\mathrm{N} \text { at risk } & 382 & 324 & 307 & 203 \\ \text { Graft survival } & 100 \% & 88.2 \% & 86.0 \% & 82.1 \%\end{array}$

FIGURE 1. A, Kaplan-Meier estimates for patient survival in different volume categories $(P=0.017)$. B, Kaplan-Meier estimates for pancreas graft survival in different volume categories $(P=0.114)$. 
TABLE 4a.

Multivariate analysis of association of risk factors with pancreas graft survival (all transplantations)

\begin{tabular}{lcc}
\hline & HR $\mathbf{( 9 5 \%} \mathbf{~ C l )}$ & $\boldsymbol{P}$ \\
\hline PDRI & $1.60(1.23-2.07)$ & $<0.001$ \\
Perfusion solution & reference & \\
$\quad$ UW & $1.28(0.95-1.72)$ & 0.111 \\
HTK & $0.71(0.09-5.40)$ & 0.739 \\
$\quad$ Other & $1.91(1.26-2.91)$ & 0.002 \\
Retransplantation & $1.04(1.00-1.07)$ & 0.024 \\
Recipient BMI & & \\
Center volume & reference & \\
$\quad$ Low volume & $0.89(0.59-1.33)$ & 0.562 \\
$\quad$ Medium volume & $0.70(0.50-0.98)$ & 0.037 \\
$\quad$ High volume & & \\
\hline
\end{tabular}

the first years of Croatian or Hungarian membership of Eurotransplant.

\section{DISCUSSION}

This study investigates the association of center volume with outcome after pancreas transplantation. We have shown that there is a significant relationship between center volume (defined as volume in 5 preceding years) and outcome, measured both in patient survival years as in pancreas graft survival years.

In this study, center volume was calculated based on the total number of pancreas transplantations in the previous 5 years. The authors have the opinion that 5 years is a reasonable timeframe to maintain an experienced program for pancreas transplantations. The calculations of volume were deliberately performed on data from preceding years, in order not to violate assumptions in analysis of longitudinal data. ${ }^{11}$ This allowed us to analyze the influence of volume on outcome, and we excluded the possibility that lower or higher volume was influenced by previous results. This is the preferred method to investigate volume-outcome in any specialty; however, results might have been clouded by the fact that centers were allowed to migrate between the categories. It could thus have been that a center was defined as medium volume in the first year, but was analyzed as being low volume in the following year. This might be considered as a limitation, but the authors consider this as a strength of the study, because this method allowed us to establish the existence of the volume-outcome relationship, without considering the individual center effect. We acknowledge the fact that center volume is a surrogate marker, because true quality depends on multiple factors, such as surgical experience, adequate recipient selection and screening, postoperative care, and long-term follow -up protocols.

Patient survival after transplantation was associated with recipient age, as well as center volume. Higher recipient age was a risk factor for patient death, whereas high center volume was a protective factor. The better patient survival might be explained by a more rigorous pretransplant screening, especially regarding cardiovascular status of the intended recipients, and more optimal posttransplant management of cardiovascular complications in higher volume centers. A recent study from Scalea et $\mathrm{al}^{15}$ demonstrated comparable patient survival in older recipients in a high volume center with very strict pretransplant cardiovascular workup.

In univariate analysis, we could not find a significant difference in graft survival and center volume. However, when correcting for relevant donor and recipient characteristics in multivariate analysis, the association with graft failure and center volume became clear. High volume centers have better results compared with low volume centers, even though they are more aggressive in their acceptance policy, indicated by higher PDRI. Furthermore, from our available recipient data, we did not establish a significant difference in transplant recipient demographics (age, BMI, waiting time, retransplantation) that could have explained these results.

Even though it is not the aim of our study, next to the volume-outcome relationship, several other factors that were significantly associated with pancreas graft survival were identified. The first is the pancreas donor risk index (PDRI), which was found to be associated with graft failure. This is in line with results from previous studies. ${ }^{16,17}$ Next to donor risk, 2 recipient factors were also found to be risk factors for inferior graft survival. Higher recipient BMI is considered a risk factor in many types of surgery, being associated with higher complication rate, and this relationship has recently been confirmed in 2 studies on pancreas transplantation. ${ }^{18,19}$ The results of our study confirm this increased risk for recipients with higher BMI. Also, retransplantation was a risk factor for graft failure (in fact, the strongest). The authors believe that this is independent of the transplant type, because we corrected for transplant type using the PDRI. Our results are in line with previously published results from a large registry analysis from the United States. ${ }^{20}$ For the subgroup of SPK transplantations, retransplantation was not a significant risk factor. This may be because of small numbers, because most retransplantations are performed in a PAK/PTA setting.

The protective effect of UW as perfusion solution in univariate analysis disappeared after multivariate analysis of all transplantations. Possible explanations could be that HTK was used in higher risk donors, retransplantations or that HTK was used more frequently in low volume centers. On the other hand, HTK was identified as an independent

\section{TABLE 4b.}

Multivariate analysis of association of risk factors with pancreas graft survival (SPK transplantations)

\begin{tabular}{lcc}
\hline & HR (95\% Cl) & $\boldsymbol{P}$ \\
\hline PDRI & $1.94(1.45-2.60)$ & $<0.001$ \\
Perfusion solution & Reference & \\
$\quad$ UW & $1.56(1.07-2.28)$ & 0.021 \\
HTK & $1.02(0.13-7.93)$ & 0.984 \\
$\quad$ Other & $1.33(0.84-2.13)$ & 0.227 \\
Retransplantation & & \\
End-stage renal disease & Reference & \\
$\quad$ Preemptive & $0.97(0.67-1.39)$ & 0.85 \\
$\quad$ Hemodialysis & $1.47(0.96-2.24)$ & 0.07 \\
$\quad$ Peritoneal dialysis & $1.04(1.00-1.07)$ & 0.047 \\
Recipient BMl & & \\
Center volume & Reference & \\
$\quad$ Low volume & $0.91(0.58-1.44)$ & 0.696 \\
Medium volume & $0.69(0.49-0.97)$ & 0.032 \\
High volume & & \\
\hline
\end{tabular}


risk factor for graft failure in the subgroup analysis of SPK transplantations. The authors think that this study provides more evidence regarding the optimal cold storage solution for pancreata. ${ }^{21}$ To identify an association was not within the scope of this study and to adequately investigate the relationship between outcome and perfusion solution a randomized controlled trial would be preferred. No association with transplant year and graft survival was found in this study, indicating that in this cohort, the era effect was of minor importance. The relatively modern cohort (without major changes in surgical techniques, preservation methods and immunosuppression) may be the reason for this absent association.

This study had some limitations. Most important one is the definition of graft failure. Because there appears to be no consensus on the definition of pancreas graft failure, graft failure was left up to the discretion of the centers. There may be significant differences in reported survival rates, depending on the definitions. Furthermore, data on reported survival and exact numbers lost to follow-up may not be complete; this may have influenced the results. Also, Eurotransplant depends on data filled in by the donor and transplant centers. Some data were missing, however, multiple imputation has been shown to provide valid results and is an accepted technique to handle missing data. ${ }^{22,23}$ We believe that using this technique did not influence the results in any way and has provided valid estimations of the missing data. The authors realize that the volume cutoffs that were chosen are debatable, however, still feasible, when looking at centers privacy and current group sizes. It could be that, next to recipient age, patient survival was associated with factors, such as preexistent peripheral artery disease, coronary or cerebrovascular disease; however, these data were not available in this study.

In conclusion, it is a remarkable finding that almost one third of all pancreas transplantations in the Eurotransplant region are being performed in centers that had performed less than on average 5 transplantations each year in the 5 preceding years. Given the fact that the highest risk organs are transplanted in the high volume centers with good outcome, it is an interesting thought that improving experience in the pancreas transplant centers may facilitate acceptance and allow transplantation of higher risk organs and increase transplant numbers.

\section{ACKNOWLEDGMENTS}

The authors would like to gratefully acknowledge Erwin de Vries (Eurotransplant data manager) for his efforts in collecting the data. Eurotransplant transplant centers are also gratefully acknowledged for submitting the data to Eurotransplant.

\section{REFERENCES}

1. Sutherland DE, Gruessner RW, Dunn DL, et al. Lessons learned from more than 1,000 pancreas transplants at a single institution. Ann Surg. 2001;233:463-501.

2. Sollinger HW, Odorico JS, Becker YT, et al. One thousand simultaneous pancreas-kidney transplants at a single center with 22-year follow-up. Ann Surg. 2009;250:618-630.

3. Kopp WH, Verhagen MJ, Blok JJ, et al. Thirty years of pancreas transplantation at Leiden University Medical Center: long-term follow-up in a large eurotransplant center. Transplantation. 2015;99:e145-e151.

4. Samuel U. Eurotransplant Annual Report. https://www.eurotransplant.org/ cms/mediaobject.php?file =ar_2014.pdf. Published 2014. Accessed 2015.

5. Axelrod DA, Sung RS, Meyer KH, et al. Systematic evaluation of pancreas allograft quality, outcomes and geographic variation in utilization. Am J Transplant. 2010;10:837-845.

6. Finger EB, Radosevich DM, Dunn TB, et al. A composite risk model for predicting technical failure in pancreas transplantation. Am J Transplant. 2013;13:1840-1849.

7. Dutch coalition "Bruggen slaan". 2012.

8. Mandal AK, Drew N, Lapidus JA. The effect of center volume on pancreas transplant outcomes. Surgery. 2004;136:225-231.

9. Malone A, Brennan DC, Wellen J, et al. The impact of center volume on simultaneous kidney-pancreas transplantation outcomes. Am J Transplant. 2015.

10. Nijboer A, Schnitzbauer AA, Ulrich F, et al. Volume-outcome relationship in organ transplantation - a systematic review. Transplant Int. 2013;26: 185-339.

11. French B, Farjah F, Flum DR, et al. A general framework for estimating volume-outcome associations from longitudinal data. Stat Med. 2012; 31:366-382.

12. Patricia M Grambsch, Therneau T. Proportional hazards tests and diagnostics based on weighted residuals. Biometrika. 1994;81:515-526.

13. Wei LJ, Lin DY, Weissfeld L. Regression analysis of multivariate incomplete failure time data by modeling marginal distributions. J Am Stat Assoc. 1989;84.

14. Horton NJ, Lipsitz SR. Multiple imputation in practice: comparison of software packages for regression models with missing variables. Am Stat. 2001;55:244-254

15. Scalea JR, Redfield RR 3rd, Arpali E, et al. Pancreas transplantation in older patients is safe, but patient selection is paramount. Transplant Int. 2016.

16. Blok JJ, Kopp WH, Verhagen MJ, et al. The Vvalue of PDRI and P-PASS as predictors of outcome after pancreas transplantation in a large European pancreas transplantation center. Pancreas. 2015.

17. Mittal S, Lee FJ, Bradbury L, et al. Validation of the Pancreas Donor Risk Index for use in a UK population. Transplant Int. 2015.

18. Bedat B, Niclauss N, Jannot AS, et al. Impact of recipient body mass index on short-term and long-term survival of pancreatic grafts. Transplantation. 2015;99:94-99.

19. Laurence JM, Marquez MA, Bazerbachi F, et al. Optimizing pancreas transplantation outcomes in obese recipients. Transplantation. 2015;99: 1282-1287.

20. Siskind E, Maloney C, Jayaschandaran V, et al. Pancreatic retransplantation is associated with poor allograft survival. Pancreas. 2015:44.

21. Barlow AD, Hosgood SA, Nicholson ML. Current state of pancreas preservation and implications for DCD pancreas transplantation. Transplantation. 2013;95:1419-1424.

22. Sterne JA, White IR, Carlin JB, et al. Multiple imputation for missing data in epidemiological and clinical research: potential and pitfalls. BMJ. 2009; 338:b2393.

23. Eekhout I, Vet HCWd, Twisk JWR, et al. Missing data in a multi-item instrument were best handled by multiple imputation at the item score level. J Clin Epidemiol. 2013. 